\section{Case Reports in Dermatology}

\title{
Acute Erythroderma in a Patient Receiving TNF- $\alpha$-Blocking Therapy for Hidradenitis Suppurativa
}

\author{
Farida Benhadou Guillaume Hellgren Fabienne Willaert \\ Véronique del Marmol \\ Dermatology, Erasme Hospital, Université Libre de Bruxelles, Brussels, Belgium
}

\section{Keywords}

TNF- $\alpha \cdot$ Adalimumab $\cdot$ Hidradenitis suppurativa $\cdot$ Psoriasis $\cdot$ Erythroderma

\begin{abstract}
Tumor necrosis factor- $\alpha$ (TNF- $\alpha$ ) normally binds to TNF- $\alpha$ receptors, leading to the inflammatory response of autoimmune diseases. Adalimumab is a TNF-inhibiting, anti-inflammatory, biological medication which binds to TNF- $\alpha$, thus reducing this inflammatory response. The use of TNF- $\alpha$-inhibiting medication, such as adalimumab, being the first FDA-approved treatment for hidradenitis suppurativa, has drastically changed the management of dermatological diseases. One rarely reported manifestation that occurs as a side effect associated with the use of TNF- $\alpha$-blocking agents is erythroderma. This study, for the first time, reports the case of a patient suffering from hidradenitis suppurativa with concomitant psoriasis, who developed a severe and acute erythrodermic rash after the start of adalimumab therapy.
\end{abstract}

\section{Introduction}

Various treatments targeting pro-inflammatory cytokines, such as tumor necrosis factor- $\alpha$ (TNF- $\alpha$ ), have changed the way cutaneous inflammatory diseases (which are the most common issues in dermatology) are managed by medical therapy $[1,2]$. The treatment of 
one of the most frequently encountered inflammatory skin conditions (psoriasis) shows the effectiveness of the TNF- $\alpha$ inhibitors. Moreover, their ability to modulate cardiovascular events in patients suffering from such conditions makes them an attractive therapeutic option for selected patients [3]. Hidradenitis suppurativa is another chronic debilitating skin condition that usually presents after puberty with painful, deep-seated, inflamed lesions in the apocrine gland-bearing areas of the body, most commonly the axillary, inguinal, and anogenital region (Dessau definition, first International Conference on Hidradenitis Suppurativa, March 30 to April 1, 2006, Dessau, Germany). It is important to note that hidradenitis suppurativa patients have high levels of TNF- $\alpha$ in their blood and in skin lesions [4]. However, a widely accepted therapeutic measure proposed for the treatment of hidradenitis suppurativa is adalimumab, a fully human anti-TNF- $\alpha$ monoclonal antibody and the first FDAapproved TNF- $\alpha$-blocking agent [5-7].

In terms of dermatological safety, TNF- $\alpha$ inhibitor use has a reassuring profile, and a few frequently reported side effects, such as respiratory, urinary, and skin infections, are reported. However, TNF- $\alpha$ blockers are contraindicated in patients with moderate to severe congestive heart failure (NYHA class III/IV). TNF- $\alpha$ blockers have also been associated with reactivation of hepatitis B virus infection in patients who are chronic carriers. Also, there are previous documentations of paradoxical reactions which include psoriasiform dermatitis, neutrophilic dermatoses, and vasculitis [7]. Erythrodermic rash, also named erythroderma, occurring after TNF- $\alpha$ inhibitors is rarely observed. Erythroderma is associated with intense and widespread reddening of the skin due to inflammatory skin disease and is often associated with exfoliation. Erythroderma is a rare entity and occurs mostly in patients having a pre-existing skin condition (e.g., psoriasis or atopic dermatitis). Thirty percent of cases of erythroderma are idiopathic, but it may also rarely be associated with a drug allergic reaction or with systemic disease (hematological or internal malignancies). Its pathogenesis is complicated, resulting in an abnormal proliferation of the keratinocytes and the release of a huge amount of pro-inflammatory cytokines in the skin [8].

Dunst-Huemer et al. [9] reported the first case of a generalized erythroderma with palmoplantar keratosis developing 5 weeks after the start of adalimumab for the treatment of psoriasis. In a recent study, El-Azhary et al. [10] reported 2 cases of exfoliative erythroderma developing within 2 weeks of introduction of TNF- $\alpha$ inhibitors for the treatment of psoriasis. One patient was receiving adalimumab and the other was treated by infliximab. In all cases, the condition was fully resolved after the discontinuation of the therapy.

To the best of our knowledge, this study reports the first case of a patient who experienced a severe generalized erythroderma 1 week after the introduction of adalimumab prescribed to treat her hidradenitis suppurativa lesions.

\section{Clinical Case}

The reference patient was a 55-year-old female diagnosed with hidradenitis suppurativa since puberty with backslides of inflammatory boils and abscesses primarily occurring in the axillary and pubic territory. The disease was classified as Hurley stage 2. Prior to diagnosis and treatment, the patient had been taking various antibiotics for many years with no effective outcomes; after that, the patient ceased all pharmaceuticals 3 years before the present diagnosis and treatment. Historically, the patient is a substantial smoker with a body mass index of $22.3 \mathrm{~kg} / \mathrm{m}^{2}$. The patient is also suffering from a mild plaque-type psoriasis, 
mainly located on the trunk and the elbows. We chose to start adalimumab to treat her hidradenitis suppurativa. She began with a high loading dosage of $160 \mathrm{mg}$.

One week after treatment, the patient developed a generalized erythrodermic rash with swollen joints on the hands and ankles without arthralgia. She also experienced a generalized myalgia and peaks of fever. The mucosae were not involved. Adalimumab was the only therapy received by the patient, and she did not take any other concomitant medication during the week preceding the rash. A skin biopsy was performed showing an unspecific picture with mild spongiosis and mild dermal immune infiltrate (Fig. 1). The blood analysis was normal. Adalimumab was instantly halted, and the rash and symptoms totally vanished after 2 weeks (Fig. 2, Fig. 3). Four months after the withdrawal of adalimumab, no new systemic therapy was introduced and the patient did not experience any new onset of erythrodermic rash or relapse of her hidradenitis suppurativa lesions. She developed mild erythematous psoriasis plaques on the knees and elbows that were successfully treated by topical steroids. We regarded the reintroduction of adalimumab as contraindicated in this patient.

\section{Discussion}

One of the greatest landmarks in the history of dermatology is the advent of biotherapies, such as TNF- $\alpha$ blockers, which have immensely improved the outcomes of inflammatory skin diseases and are usually well tolerated. However, there are only a few reported severe side effects associated with their use in dermatological therapies, and the majority of side effects are mild and are mainly represented by infections of the respiratory or urinary tract and of the skin. An immune-mediated class of cutaneous side effects has also been reported.

Such immune-mediated responses include paradoxical reactions, usually defined as beginning or worsening of certain dermatoses, such as psoriasis or hidradenitis suppurativa, which have originally been treated using the TNF- $\alpha$ inhibitors. Other immune skin disorders, such as dermatomyositis, lupus-like syndrome, vasculitis, granuloma annulare, sarcoidosis, alopecia areata, vitiligo, lichen planus, bullous dermatoses, allergic reactions (hypersensitivity, urticaria, and angioedema), erythema multiforme, Stevens-Johnson syndrome, and toxic epidermal necrolysis, among others, have been reported [8].

It is important to note that the few and rarely recorded allergic reactions to biotherapies, though with an unknown peak of incidence, are in so many respects different from those triggered by other types of drugs, since they are highly immunogenic and act on the immune system. Allergic reactions to TNF- $\alpha$ inhibitors are also different in their clinical expression based on the specific disease for which they were prescribed. However, Pichler [11] proposed a new method of grading these allergic reactions by grouping them into 5 types $(\alpha$, $\beta, \delta, \varepsilon$, and $\gamma$ type). With respect to Pichler's method, we can, therefore, classify our clinical case as $\alpha$ type reaction, which occurred as a result of an increased level of cytokines in the system, which may cause the patient to experience fever, fatigue, myalgia, generalized rash, and gastrointestinal symptoms and can also, but rarely, result in multi-organ dysfunction.

In the event of adalimumab injection, there have been reported cases of mild reactions at the injection site in about $20 \%$ of patients under treatment for hidradenitis suppurativa, with less than $1 \%$ of reports on allergic rash cases, which are rarely associated with urticaria and angioedema [12-14]. In particular, this study reports the uncommon case of a reaction experienced in the form of an erythrodermic rash by a hidradenitis suppurativa patient treated with adalimumab injection. This reaction was accompanied by systemic symptoms at 
about 1 week of discontinuation. However, the patient refused to perform an allergic skin test with adalimumab. We can conclude that she built up a cutaneous immune-mediated reaction to adalimumab accompanied by systemic symptoms, classified as type $\alpha$ according to the classification of Pichler [11].

In consideration of the circumstances, we do have certain doubts as to whether to classify the side effect as a paradoxical reaction (exacerbation of her psoriasis) or as a pure allergic reaction to adalimumab. Dunst-Huemer et al. [9] and El-Azhary et al. [10] have also reported 3 cases of severe generalized erythroderma in psoriasis patients undergoing treatment with TNF- $\alpha$ inhibitors, and their final diagnosis was the exacerbation of existing psoriasis, but all authors regarded the reintroduction of TNF- $\alpha$ inhibitors as contraindicated. The patient reported by Dunst-Huemer et al. [9] developed an erythrodermic rash 5 weeks after the start of adalimumab, and no other concomitant medication was taken during the period preceding the rash. The rash cleared after the use of topical steroids and the start of ustekinumab therapy. The cases reported by El-Azhary et al. [10] developed an erythrodermic rash within a short period after the start of adalimumab and infliximab, respectively. No concomitant medication was taken during the period preceding the rash. After the introduction of ustekinumab therapy, the rash completely regressed in the first patient. The second patient experienced a moderate improvement after acitretin introduction, but the authors do not document the long-term follow-up in detail.

One objective of our work was to highlight this rare side effect for dermatologists and to help them to properly consider the management of hidradenitis suppurativa patients using adalimumab therapy. This is because the dose regimen is higher than for psoriasis, including a loading dose of $160 \mathrm{mg}$ followed by a weekly dosage of $40 \mathrm{mg}$. Therefore, there is a high risk of paradoxical reactions, especially in patients who may have another known inflammatory disease, and this has to be taken into consideration. It would be interesting to consider the blood antidrug antibody levels in these patients.

The pure allergic reaction to adalimumab or other TNF- $\alpha$ inhibitors can be tested by a skin test (prick and intradermal injection); however, as described by Benucci et al. [15], the tests can be negative. Additionally, more studies are required in terms of a detailed investigation of registries to help dermatologists to better understand and properly classify responses that occur after introducing TNF- $\alpha$ inhibitors as a therapeutic measure. Finally, we emphasize the crucial need of well-constructed hypersensitivity screening tests in our daily practice to avoid an undocumented and inappropriate withdrawal of the medication and to guide the dermatologist in applying a precise therapeutic procedure.

\section{Statement of Ethics}

The patient provided consent for publication.

\section{Disclosure Statement}

The authors have declared that no competing interests exist. 


\section{Case Reports in Dermatology}

\begin{tabular}{l|l}
\hline Case Rep Dermatol 2018;10:7-12 \\
\hline DOI: 10.1159/000485911. & $\begin{array}{l}\text { (c) 2018 The Author(s). Published by S. Karger AG, Basel } \\
\text { www.karger.com/cde }\end{array}$ \\
\hline
\end{tabular}

Benhadou et al.: Acute Erythroderma in a Patient Receiving TNF- $\alpha$-Blocking Therapy for Hidradenitis Suppurativa

\section{References}

1 Cordoro KM, Feldman SR: TNF-alpha inhibitors in dermatology. Skin Therapy Lett 2007;12:4-6.

2 Katugampola RP, Lewis VJ, Finlay AY: The Dermatology Life Quality Index: assessing the efficacy of biological therapies for psoriasis. Br J Dermatol 2007;156:945-950.

-3 Parisi R, Symmons DPM, Griffiths CEM, Ashcroft DM; Identification and Management of Psoriasis and Associated Comorbidity (IMPACT) project team: Global epidemiology of psoriasis: a systematic review of incidence and prevalence. J Invest Dermatol 2013;133:377-385.

4 Matusiak L, Bieniek A, Szepietowski JC: Increased serum tumour necrosis factor-alpha in hidradenitis suppurativa patients: is there a basis for treatment with anti-tumour necrosis factor-alpha agents? Acta Derm Venereol 2009;89:601-603.

-5 Kim ES, Garnock-Jones KP, Keam SJ: Adalimumab: a review in hidradenitis suppurativa. Am J Clin Dermatol 2016;17:545-552.

6 Gupta AK, Studholme C: Adalimumab (Humira) for the treatment of hidradenitis suppurativa. Skin Therapy Lett 2016;21:1-4.

7 Kimball A, et al: Two phase 3 trials of adalimumab for hidradenitis suppurativa. N Engl J Med 2016;375:422-434.

-8 César A, Cruz M, Mota A, Azevedo F: Erythroderma. A clinical and etiological study of 103 patients. J Dermatol Case Rep 2016;10:1-9.

$\$$ Dunst-Huemer KM, Scheurecker C, Auboeck J: Generalized erythroderma and palmoplantar hyperkeratosis in a patient receiving TNF-alpha antagonist therapy. J Cutan Pathol 2013;40:855-856

10 El-Azhary RA, Lehman JS: Exfoliative erythroderma after tumor necrosis factor- $\alpha$ inhibitor therapy. Int Dermatol 2016;55:e214-e215.

11 Pichler WJ: Adverse side effects to biological agents. Allergy 2006;61:912-920.

12 Corominas M, Gastaminza G, Lobera M: Hypersensitivity reactions to biological drugs. J Investig Allergol Clin Immunol 2014;24:212-225.

-13 Borras-Blasco J, Navarro-Ruiz A, Borras C, Castera E: Adverse cutaneous reactions induced by TNFalpha antagonist therapy. South Med J 2009;102:1133-1140.

14 Mallo S, Santos-Juanes J: Adalimumab-induced urticaria. Actas Dermosifiliogr 2007;98:511-512.

15 Benucci M, Manfredi M, Demoly P, Campi P: Injection site reactions to TNF-alpha blocking agents with positive skin tests. Allergy 2008;63:138-139.

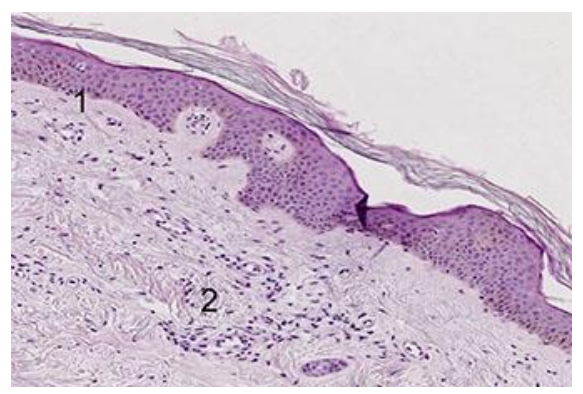

Fig. 1. Skin biopsy performed during the erythrodermic rash showing an unspecific picture of mild spongiosis (1) and mild dermal inflammatory infiltrate (2). 


\section{Case Reports in Dermatology}

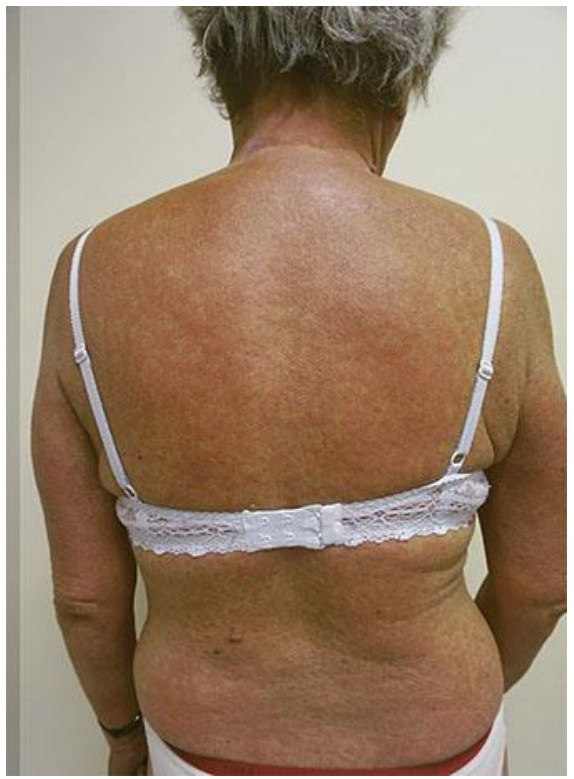

Fig. 2. Papular erythrodermic rash on the trunk 1 week after the initiation of adalimumab.

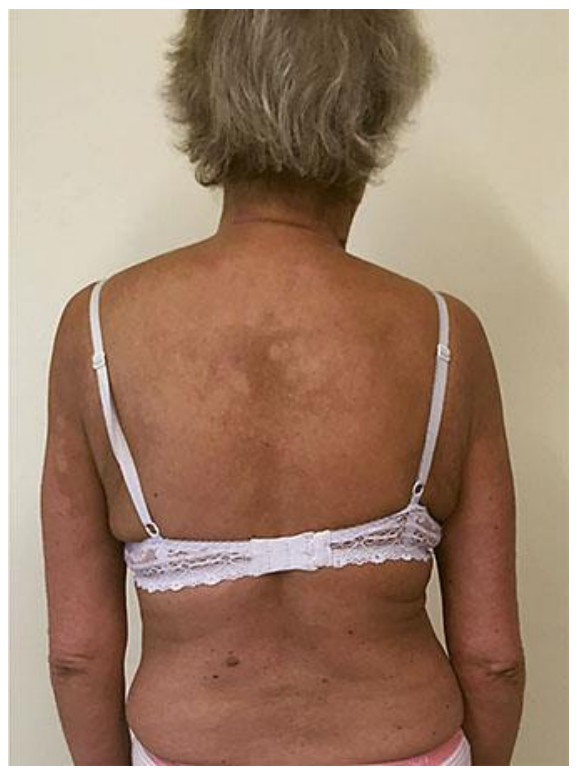

Fig. 3. Clearance of the erythrodermic rash with remaining postinflammatory skin spots 2 weeks after the withdrawal of adalimumab. 